Chronique des activités archéologiques de

\title{
Recherches sur les terres cuites votives de Medma
}

Étude du matériel des fouilles Orsi (1912-1913) du site de Calderazzo

(Rosarno, Calabre). Campagnes 2017-2019.

Ágnes Bencze

\section{Q OpenEdition}

\section{Journals}

\section{Édition électronique}

URL : http://journals.openedition.org/cefr/3947

DOI : $10.4000 /$ cefr.3947

ISSN : 2282-5703

Éditeur

École française de Rome

\section{Référence électronique}

Ágnes Bencze, "Recherches sur les terres cuites votives de Medma », Chronique des activités archéologiques de l'École française de Rome [En ligne], Italie du Sud, mis en ligne le 29 novembre 2019, consulté le 03 décembre 2019. URL : http://journals.openedition.org/cefr/3947 ; DOI : 10.4000/cefr. 3947

Ce document a été généré automatiquement le 3 décembre 2019.

(c) École française de Rome 


\title{
Recherches sur les terres cuites votives de Medma
}

\author{
Étude du matériel des fouilles Orsi (1912-1913) du site de Calderazzo \\ (Rosarno, Calabre). Campagnes 2017-2019.
}

\section{Ágnes Bencze}

\section{NOTE DE L'AUTEUR}

Les recherches résumées ci-dessous se sont déroulées dans le cadre d'un projet soutenu par l'École française de Rome, l'Université Catholique Péter Pázmány de Budapest et le Musée des Beaux-Arts de Budapest, en collaboration avec le Musée Archéologique National de Reggio Calabria et la Surintendance ABAP responsable pour les territoires de Reggio Calabria et Vibo Valentia (Calabre). Du côté italien, le projet a profité du soutien de Carmelo G. Malacrino, directeur du Musée Archéologique National de Reggio Calabria, et de Fabrizio Sudano, inspecteur de la Surintendance.

L'équipe dirigée par Ágnes Bencze (Université Catholique Péter Pázmány) comprenait durant les campagnes de 2017, 2018 et 2019 les participants suivants : Attila Hajdú (doctorant, Université de Szeged), Noémi Tóth (assistant archéologue, Musée des Beaux-Arts de Budapest), Zita Rostás (restauratrice, Musée des Beaux-Arts de Budapest), Anna Meszleny, Ádám Hegedűs, Veronika Berek, Linda Tarr, Judit Vörös et Dániel János Széll (étudiants à l'Université Péter Pázmány), Adél Ternovácz, Bence Párkányi (étudiants à l'Université Eötvös Loránd de Budapest) et Luca Pintaudi (étudiant de spécialisation à l'Università del Sacro Cuore, Milan).

En outre, le travail de l'équipe a profité des visites de consultation des experts suivants : en 2017 Maurizio Paoletti (Università della Calabria, Cosenza), en 2018 Arianna Esposito (Université de Bourgogne, Dijon) et Claudio Sabbione (ancien inspecteur de la Surintendance Archéologique de la Calabre), en 2019 Franco Prampolini (Università di Reggio Calabria, faculté d'Architecture), Ermanno A. Arslan (Accademia Nazionale dei Lincei) et Antonella Pautasso (CNR Catania). 
1 L'unité culturelle de Locres Epizéphyrienne et ses colonies fondées sur la côte tyrrhénienne de la Calabre actuelle, Medma et Hipponion, est un fait généralement accepté dans la bibliographie, au moins en ce qui concerne le $\mathrm{VI}^{\mathrm{e}}$ et la première moitié $\mathrm{du} \mathrm{V}^{\mathrm{e}}$ siècle av.J-C. ${ }^{1}$. Les sanctuaires de l'aire locrienne ont produit de grandes quantités d'objets votifs datant de cette période, parmi lesquels les terres cuites constituent la classe dominante. Outre les célèbres pinakes, les sites cultuels ont livré aussi des milliers de fragments de statuettes en ronde-bosse. Depuis les premières découvertes publiées jusqu'à nos jours, ces grandes productions, visiblement entrecroisées, ont toujours suscité de l'intérêt dans la recherche. Malgré cela, les tentatives de documentation et de classement n'ont été qu'occasionnelles et limitées, principalement à cause des difficultés techniques liées à l'accessibilité du matériel. Notre projet a pour but immédiat la documentation et la classification systématique des terres cuites votives figurées, découvertes avant 2000 sur le site nommé Calderazzo, en bordure septentrionale de la ville moderne de Rosarno (fig. 1). Sur le long terme, nous envisageons d'établir une documentation suffisante pour une étude approfondie portant sur les connexions entre les productions coroplastiques des trois cités de la sphère locrienne, qui permettra de mieux comprendre le fonctionnement de leurs sanctuaires, les pratiques rituelles qui y étaient accomplies, les choix artistiques de leurs communautés, ainsi que leurs liens culturels².

Fig. 1 - Vue aérienne du site Calderazzo (Rosarno).

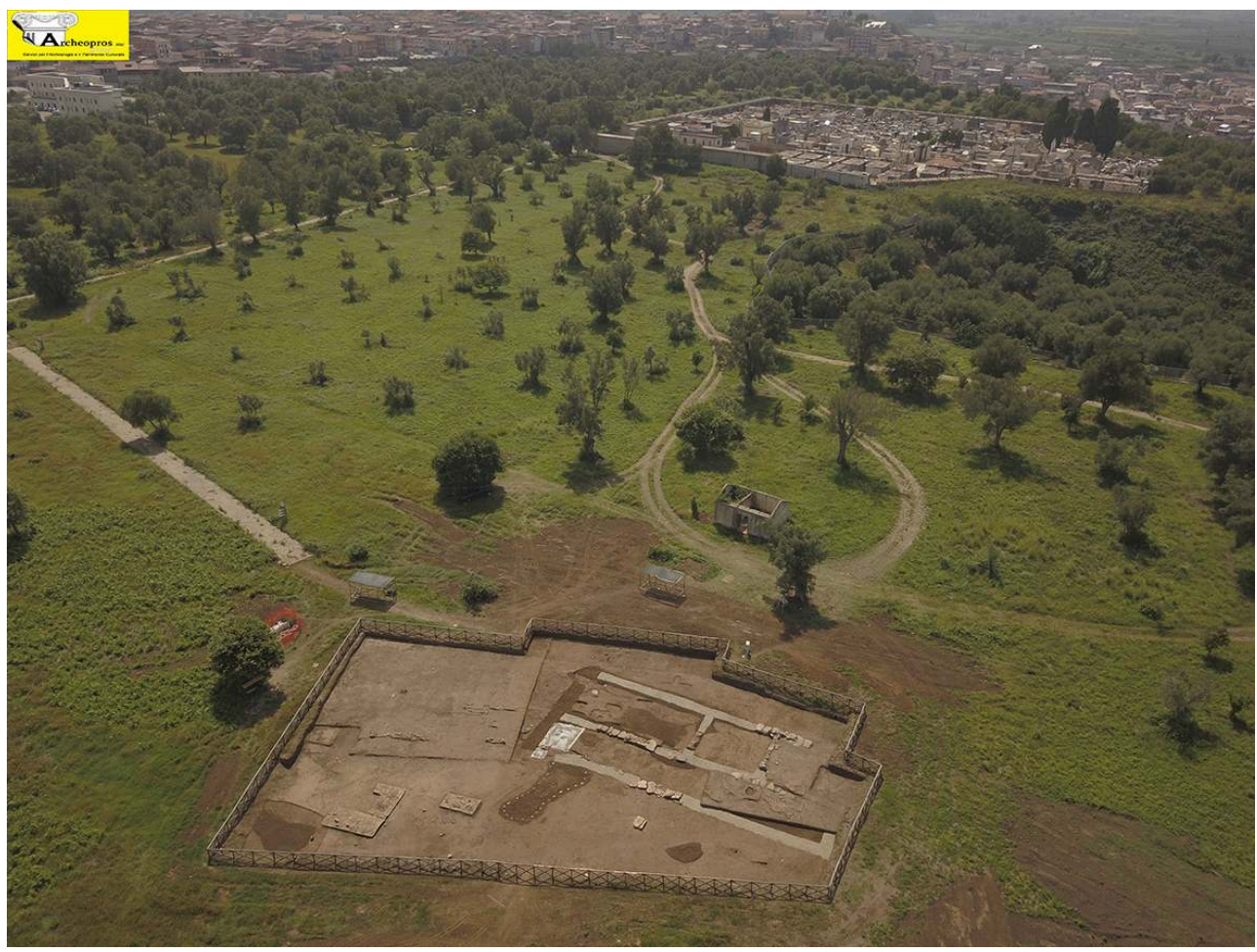

Crédit : Archeopros.

2 Au cours des trois campagnes, qui se sont déroulées de 2017 à 2019 au mois de juillet, notre équipe a commencé et poursuivi le travail de documentation sur deux sites : au Musée National de Reggio Calabria et dans les réserves de la Surintendance ABAP - RC$\mathrm{VV}$, situées à Rosarno. Les trois campagnes nous ont permis de dresser un cadre 
d'ensemble du matériel provenant des fouilles de Paolo Orsi conduites sur le site Calderazzo ${ }^{3}$, et de documenter au total 1089 pièces.

\section{Conditions des découvertes et problèmes}

3 Le compte rendu publié par Paolo Orsi dans les suppléments des Notizie degli Scavi di Antichità de 1913 est resté pendant plusieurs décennies la seule publication générale des découvertes de Rosarno/Medma et spécifiquement du sanctuaire de Calderazzo, et ce en dépit des intentions originaires de l'auteur qui n'y voyait qu'un rapport préliminaire à une publication exhaustive $e^{4}$. Tandis que les fouilles conduites de temps à autre sur le site durant les cent dernières années ont apporté des précisions utiles pour la topographie du sanctuaire ${ }^{5}$, l'amas imposant des objets votifs n'a pas été abordé systématiquement depuis 1913. La grande favissa rituelle, source principale de la documentation coroplastique du sanctuaire de Calderazzo, mesure au moins $33 \mathrm{~m}$ de long et $3,5 \mathrm{~m}$ de large, tandis que sa profondeur variait de 2 à $3 \mathrm{~m}^{6}$. La quantité de statuettes (rarement complètes) et de fragments de statuettes et reliefs qui y furent ensevelis reste encore à déterminer aujourd'hui.

Malgré l'intérêt général, motivé par la qualité technique et artistique exceptionnelle des statuettes de Medma, qu'on a pu observer dans la littérature consacrée à l'art de la Grande Grèce au cours des décennies passées, il n'y a eu que deux tentatives d'étude systématique de ce matériel : celle de Rebecca Miller au début des années $1980^{7}$ et celle annoncée par Daphni Doepner en $2007^{8}$. Ces deux tentatives étaient toutefois destinées à rester limitées et partielles ${ }^{9}$, surtout en raison des conditions particulières dans lesquelles se trouve cette documentation importante, à cause des vicissitudes qu'elle a connu depuis sa découverte. En fait, une partie consistante du matériel coroplastique provenant des fouilles d'Orsi ne fut ni nettoyé ni inventorié lors de la découverte. Grâce à une première sélection, quelques centaines de pièces furent néanmoins choisies pour la publication préliminaire et sans doute pour des expositions, et furent soigneusement restaurées; une partie de celles-ci se trouvent aujourd'hui dans les expositions permanentes du Musée National de Reggio Calabria et du petit Musée de Rosarno, tandis que beaucoup d'autres sont retournées dans les réserves. De plus, au cours des cent dernières années, le lieu de conservation des terres cuites de Rosarno a changé plusieurs fois, et elles sont actuellement partagées entre le Musée National et le muséedépôt de la Surintendance, situé à Rosarno. Tandis que la plupart des fragments arrivés à Reggio Calabria sont pourvus de numéros d'inventaire et montrent les traces de nettoyage voire parfois de restauration, le dépôt de Rosarno abrite aussi des lots de matériel provenant des anciennes fouilles d'Orsi, mais apparemment intacts.

Compte tenu de ces conditions, qui impliquent également que le nombre précis des objets intéressés par la recherche reste à déterminer, notre projet a été programmé dès le début comme un travail en équipe, permettant de mener une documentation systématique et en parallèle dans les deux lieux de conservation. Suivant les accords pris avec la direction du Musée National de Reggio Calabria, notre équipe s'est chargée aussi de la révision des anciens inventaires et d'insérer les objets étudiés dans le nouveau système d'inventaire du Musée (fig. 2-3). 
Fig. 2 - Travail de documentation dans les bureaux du Musée National Archéologique de Reggio Calabria.

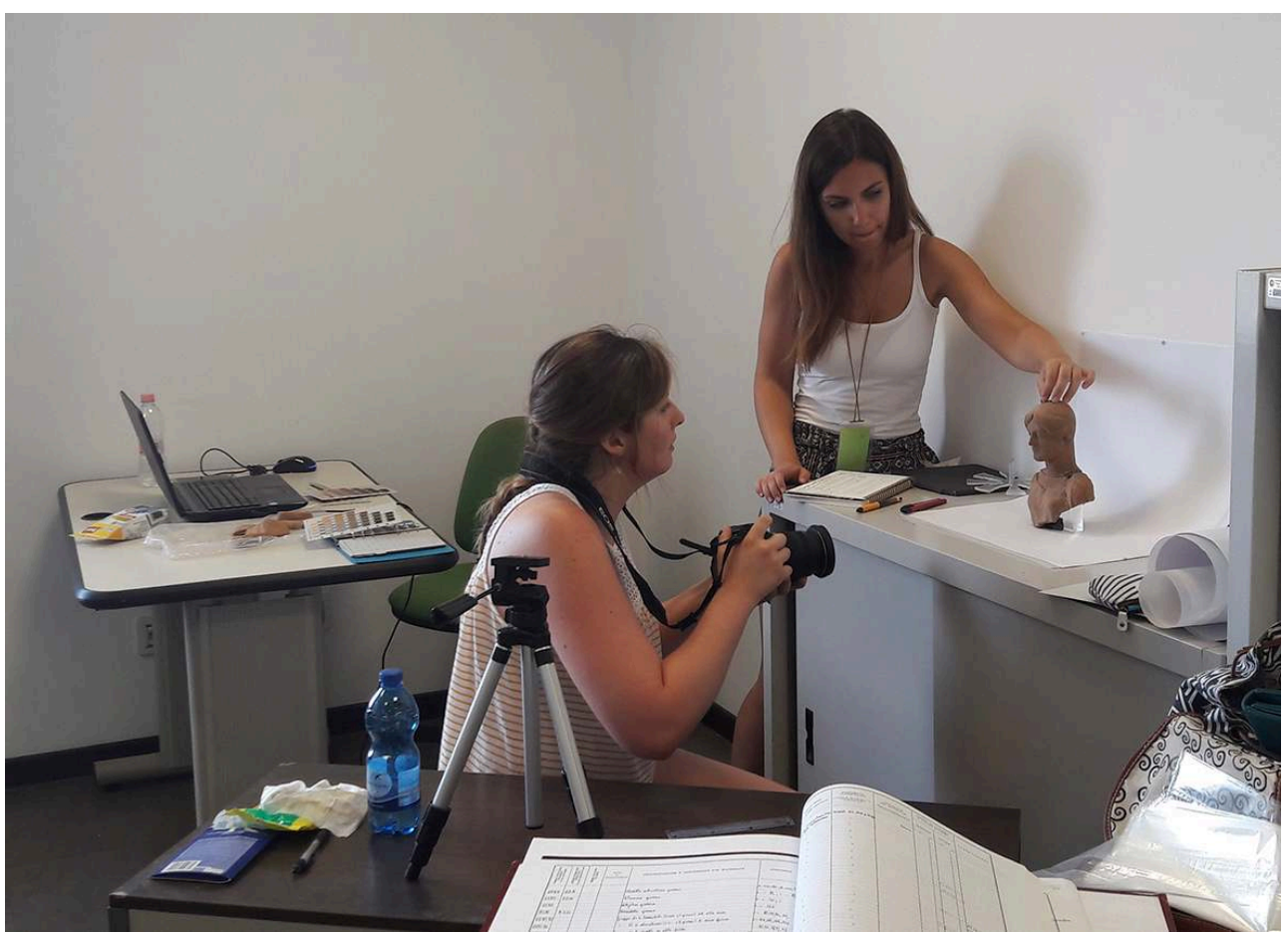

Crédit : Á. Bencze.

Fig. 3 - Travail de documentation dans les bureaux du Musée National Archéologique de Reggio Calabria.

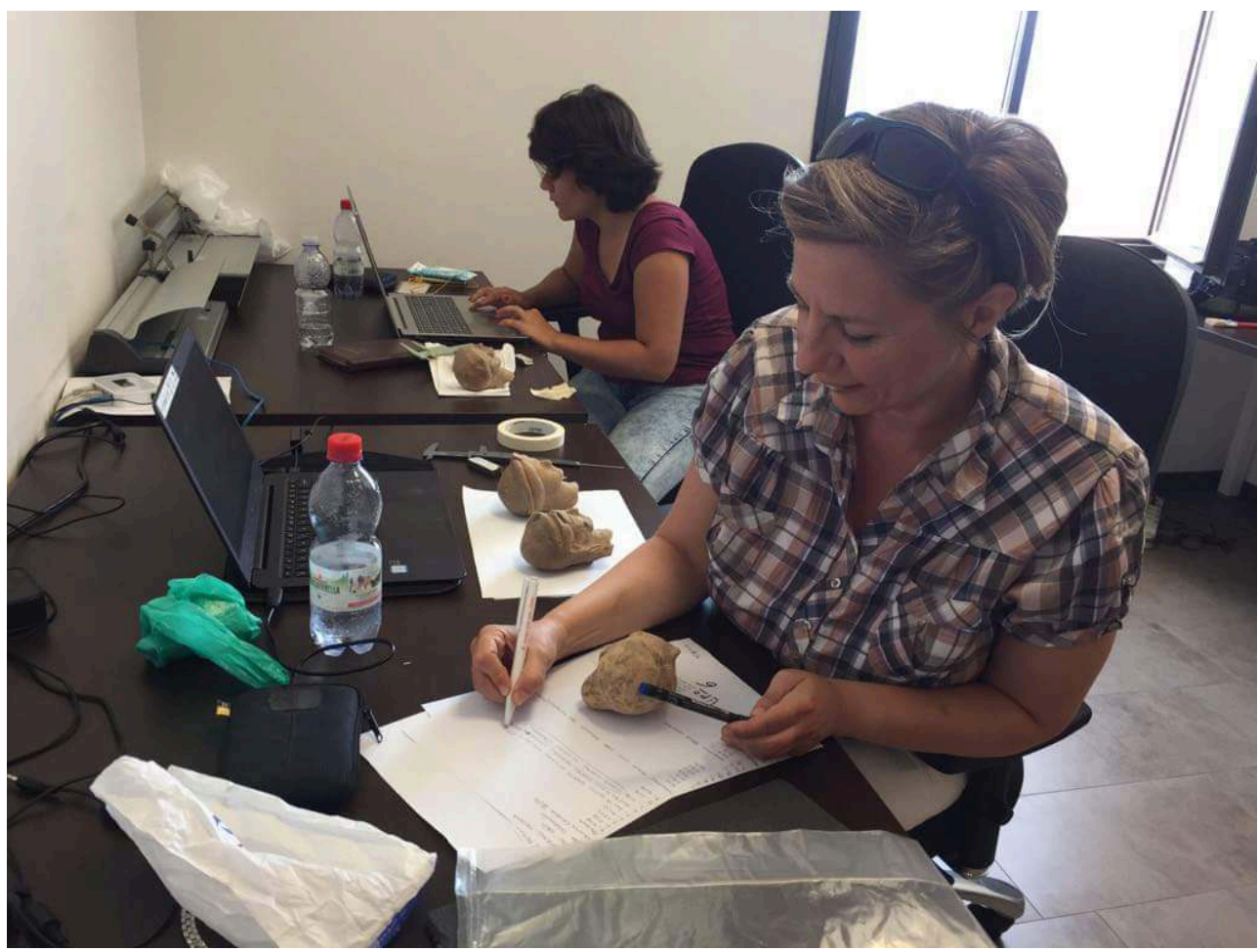

Crédit : Á. Hegedűs. 
6 de façon aléatoire, il n'est plus possible de remonter aux contextes ou à la structure des dépôts votifs antiques, excluant donc toute étude reposant sur ceux-ci. Tout en reconnaissant la possibilité que notre étude puisse un jour bénéficier des résultats d'éventuelles recherches consacrées à la topographie et aux structures du dépôt votif de Calderazzo ${ }^{10}$, il est évident que pour les objectifs envisagés par ce projet, le matériel doit être interprété en lui-même, dans son état actuel.

7 Notre objectif premier consiste à la reconstruction la plus complète possible de la typologie coroplastique présente dans le sanctuaire de Calderazzo. Afin de permettre ce travail de classement, une base de données est développée qui intègre tous les objets provenant de la favissa Orsi, y compris aussi les fragments les moins informatifs. Cette documentation constitue, en fait, le seul moyen pour formuler des hypothèses statistiques, voire pour parvenir à déterminer à l'avenir la provenance des terres cuites, possiblement à l'aide d'analyses géochimiques, et pour reconstruire les typologies, y compris les séquences et les jeux de moules (fig. 4-6). Si l'on ne tient compte que des objets provenant des fouilles de Calderazzo et, plus précisément, de la favissa, les cadres chronologiques de la production semblent se limiter entre l'époque archaïque avancée (à peu près les décennies centrales $\mathrm{du} \mathrm{VI}^{\mathrm{e}}$ siècle) et la seconde moitié $\mathrm{du} \mathrm{V}^{\mathrm{e}}$ siècle av. J.-C. Il nous reste encore à déterminer précisément les limites finales des dépositions de terres cuites dans ce sanctuaire et le rapport de cette production avec la suite, c'est-à-dire avec la coroplathie de Medma d'époque classique avancée, qui est bien attestée par les complexes votifs des autres lieux de culte de la cité et par les trouvailles de la nécropole ${ }^{11}$. Quant au problème des débuts de la production de Medma, la question la plus importante à éclaircir sera celle des liens avec les communautés des deux autres cités de la « sphère Locrienne »-parmi lesquelles Hipponion ne semble pas être moins importante que Locres elle-même -, et ce tant du point de vue rituel que de la production artisanale. 
Fig. 4 - Rosarno, Musée Archéologique de la Surintendance, 2017.

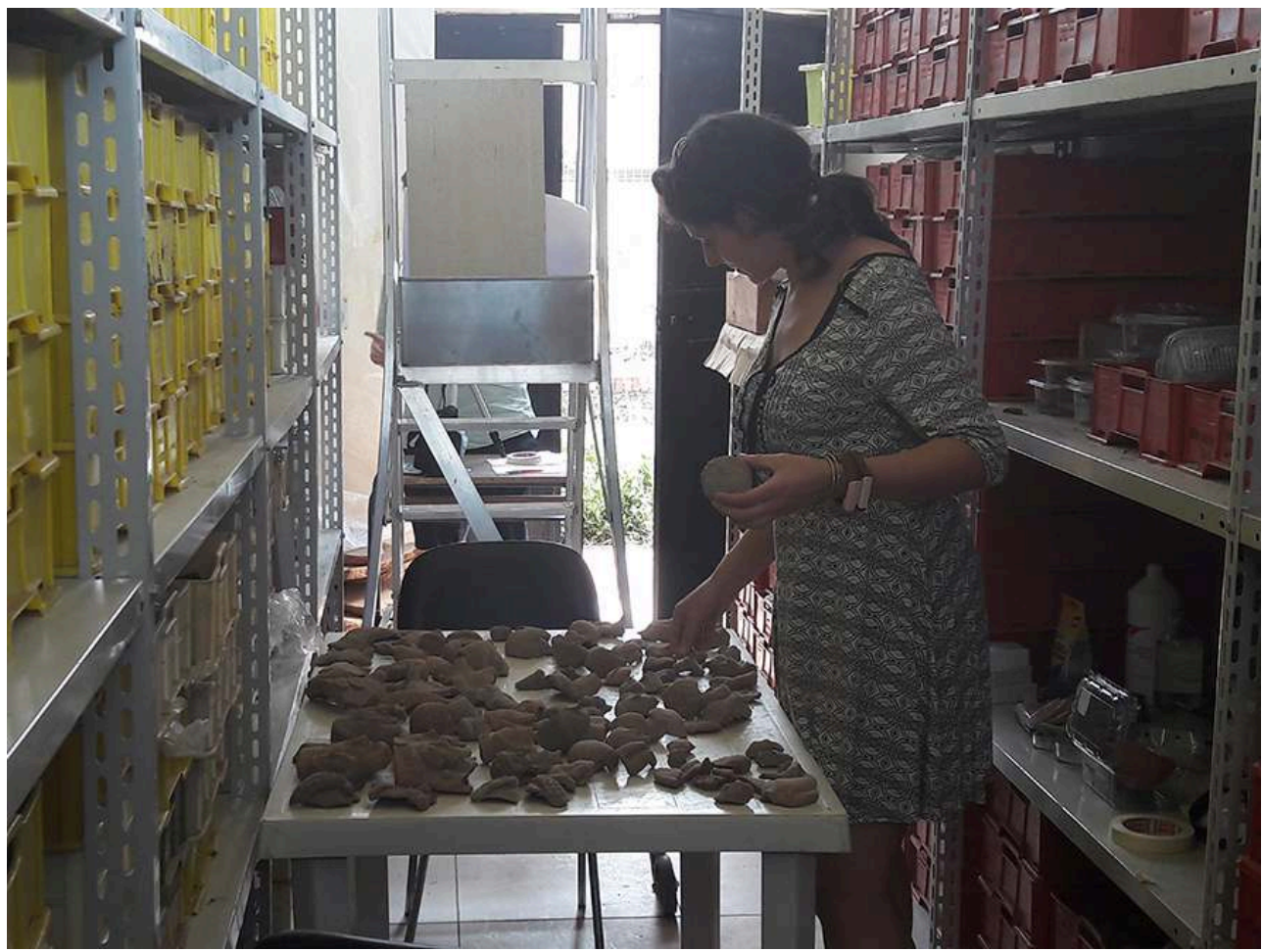

Crédit : Á. Bencze.

Fig. 5 - Terres cuites en cours de restauration au Musée Archéologique de la Surintendance à Rosarno.

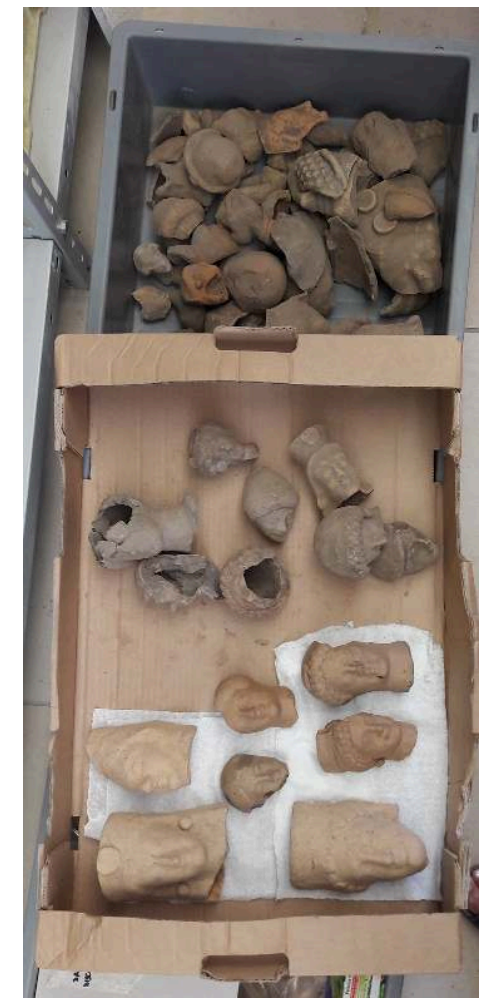

Crédit : Á. Bencze. 
Fig. 6 - Tentative de classification de fragments ornementaux au Musée Archéologique de la Surintendance à Rosarno.

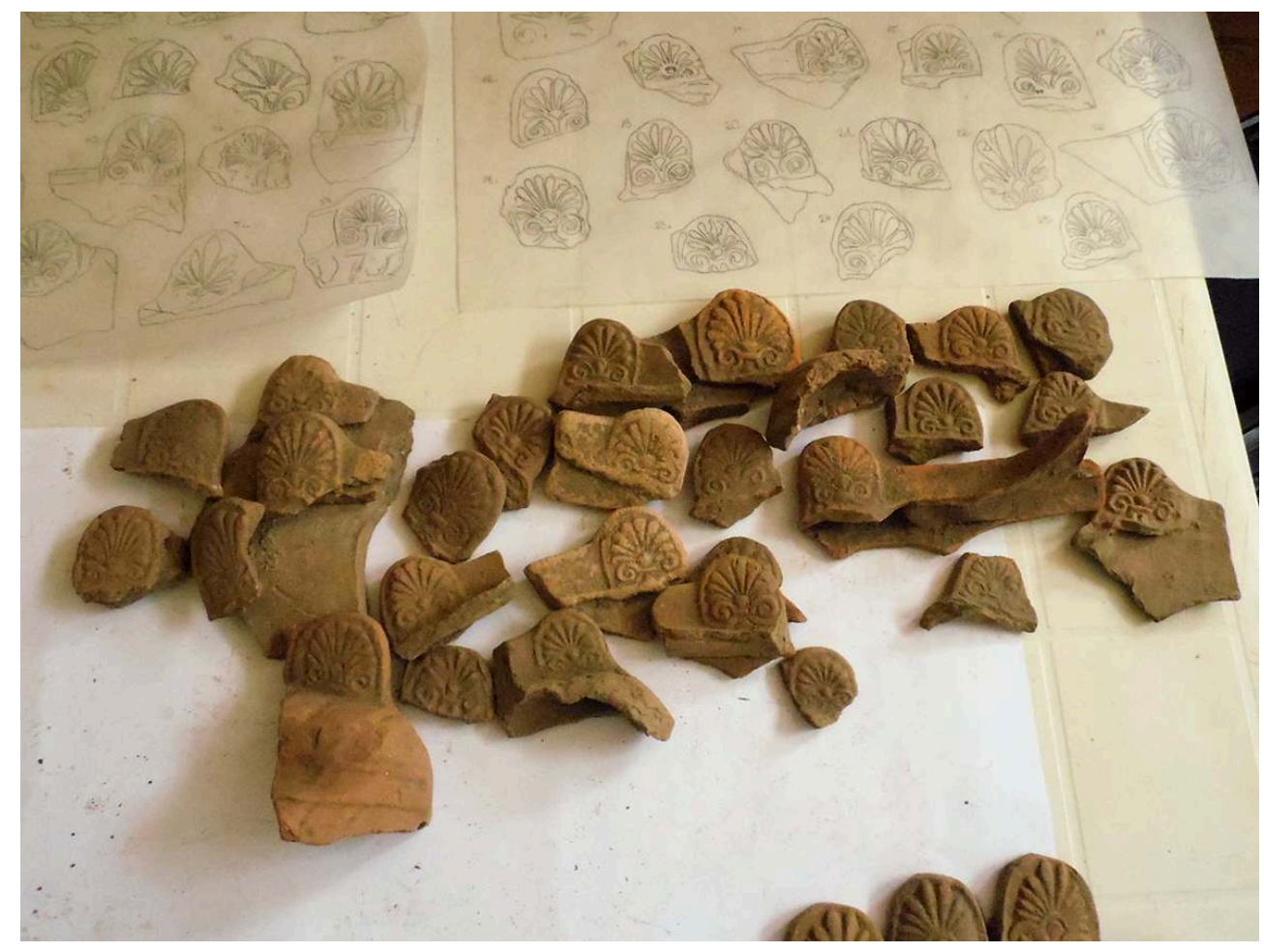

Crédit : Á. Bencze.

\section{Documentation, base de données et système typologique}

\section{Classement préliminaire des documents}

Les 1098 pièces recensées jusqu’à présent se divisent entre le Musée National de Reggio Calabria et le Musée-dépôt de Rosarno de la façon suivante : à Reggio Calabria nous avons catalogué 590 objets, c'est-à-dire la quasi-totalité du matériel conservé dans ce lieu, à l'exception des pièces en exposition et un lot de matériel en cours de restauration; à Rosarno, où le travail de documentation est précédé par la restauration exécutée par notre équipe, nous sommes arrivés à 499 exemplaires. Les réserves de Rosarno abritent encore un grand lot de terres cuites à nettoyer et restaurer avant d'être documentées, dont on peut estimer la quantité entre 500 et 600 pièces environ. $\mathrm{Au}$ sein de ce dernier groupe se trouvent certainement des statuettes fragmentaires à recomposer, un travail qui nécessitera une organisation logistique un peu différente pour la campagne programmée en 2020.

La disposition complètement aléatoire dans laquelle les objets se trouvent actuellement exclut aussi toute sorte de groupement préalable du matériel. Au contraire, la seule démarche possible consiste en la révision des unités (c'est-à-dire les boîtes organisées plus ou moins au hasard) dans l'ordre accidentel de leur disposition dans les réserves, mais visant à l'exhaustivité. Par conséquent, il serait prématuré d'anticiper des données statistiques avant de la conclusion du travail de documentation. Il semble 
toutefois utile de définir des groupes majeurs, établis sur la base de quatre types de critères, sans indiquer pour le moment leurs proportions à l'intérieur de l'ensemble, ne serait-ce que très approximativement :

10 1. Du point de vue d'une première approximation chronologique, 20 à $25 \%$ des fragments représentent une phase de production "préliminaire ", coïncidant avec la deuxième moitié du $\mathrm{VI}^{\mathrm{e}}$ siècle av. J-C. Au moins $60 \%$ correspond à la phase d'épanouissement, étroitement liée du point de vue stylistique à la production des pinakes locriens (première moitié du $\mathrm{V}^{\mathrm{e}}$ siècle av. J.-C.) ; 15 à $20 \%$ attestent la poursuite des offrandes, avec des traits stylistiques correspondant aux tendances de goût du $\mathrm{V}^{\mathrm{e}}$ siècle avancé.

11 2. Du point de vue des genres d'objets les groupes les plus importants numériquement sont les suivants :

- protomés féminines, exclusivement de style archaïque (5 à $10 \%)$;

- statuettes et bustes « xoaniques » de type archaïque (5 à $10 \%$ );

- statuettes et fragments de statuettes de figure féminine trônant ou debout ; bustes féminins stylistiquement liés aux statuettes (au moins $60 \%$ );

- types iconographiques variés, surtout masculins (5 à $10 \%)$;

- autres (pinakes, figurines modelées à la main, terres cuites représentantes des objets) (10 à $15 \%)$.

3. Les qualités d'argile présentes dans le matériel se divisent, à simple observation, en trois grands groupes, dont les caractéristiques de base sont évidentes à l'œil nu. Il existe toutefois plusieurs variantes à l'intérieur de chacun, surtout au sein du groupe III, qui apparait aussi comme le plus abondant quantitativement. Les différences observées par autopsie dépendent de la composition de la pâte, des techniques de traitement qu'elle a subies, ainsi que de la température de la cuisson. Par conséquent, le groupement et les descriptions qui suivent ci-dessus ne sont à considérer que comme préliminaires à une analyse archéométrique plus complexe, qui est envisagée pour les campagnes finales de ce projet ${ }^{12}$.

- Groupe I : argile molle, friable et poreuse, couleur jaune pâle (nuances Munsell 10YR 8/2 à 7/4), aux grains moyens, contenant du mica et peu d'autres inclusions, correspondant 5 à $10 \%$ des artefacts, tendanciellement coïncidant avec les typologies de la phase archaïque ;

- Groupe II : argile très compacte, dure, couleur pêche (Munsell 5YR 8/2 à 8/4, Munsell 7.5YR 7/4 à 7/6) sur la surface et gris vivace à l'intérieur, aux grains fins, plutôt bien dépurée, contenant surtout des grandes inclusions couleur rouille, autour desquelles la pâte tend à s'ébrécher, correspondant à 20-30\% des artefacts ;

- Groupe III : argile plutôt dure, mais poreuse, couleur rouge brique (Munsell 5YR 7/6 à 7/8 et 6/6 à 6/8), grains de finesse variable, jusqu'à des pâtes très grossières, particulièrement riche de mica et d'inclusions blanches et sombres, correspondant à la majorité des objets.

4. L'observation des qualités physiques de l'argile est complétée par celle des traces qui révèlent les techniques de fabrication utilisées par les artisans. Les données de ces deux critères sont entrecroisées pour établir des groupes d'objets appartenant probablement à des traditions communes d'atelier. La technique utilisée dans la majorité des cas est le moulage creux, à l'aide d'un moule ou de plusieurs moules de front, mais non par moules bivalves. Le revers des statuettes est toujours fermé par une paroi façonnée à la main ou à l'aide d'un outil. Les statuettes et les protomés archaïques, tout comme les statuettes et les grands bustes $\mathrm{du} \mathrm{V}^{\mathrm{e}}$ siècle sont tous décorés par des éléments ajoutés à la main, souvent remaniés. Les retouches exécutées à l'aide d'outils à pointe sur la pièce 
finie ou sur le moule sont tendanciellement la règle dans les groupes classiques et rares dans la phase première.

D'après nos observations actuelles, les cas de séquences de moules, c'est-à-dire de plusieurs "générations " appartenant à un même type coroplastique, sont très rares à Medma. Les diverses classes de dimensions semblent correspondre à des groupes de créations plastiques indépendantes. Une petite série de terres cuites de petites dimensions $(10-15 \mathrm{~cm})$, identifiée au sein du matériel, ne semble pas être directement liée aux statuettes élaborées, de dimensions moyennes $(30-40 \mathrm{~cm})^{13}$.

Contrairement à ce qui est supposé en général, la variété des terres cuites de Medma n'est pas le résultat de retouches et remodelages constants sur un nombre limité de types coroplastiques, mais reflète une richesse particulière de créations plastiques autonomes, produites pour la plupart parallèlement au cours de quelques décennies.

\section{Méthode de classement et critères de description}

16 Compte tenu des spécificités résumées ci-dessus, qui caractérisent cet ensemble de documents coroplastiques, l'objectif primaire du projet consiste dans l'établissement du répertoire des types, c'est-à-dire des groupes d'objet reproduisant mécaniquement des créations plastiques indépendantes de base. Il est d'ores et déjà établi que le nombre de ces créations plastiques est supérieur à une centaine. La définition d'un type ne repose pas sur une description iconographique, puisque les attributs iconographiques étaient les éléments les plus susceptibles de changer d'une reproduction à l'autre, par le biais des ajouts et remodelages secondaires. Ainsi, la méthode choisie consiste dans l'analyse stylistique des exemplaires documentés, qui permet aussi de discerner les déformations, intentionnelles ou non, apportées sur la pièce au cours de la fabrication et, compte tenu de celles-ci, d'arriver à établir la création plastique correspondant au « prototype».

17 L'étape suivante consiste à reconnaître les connexions entre les types identifiés. La première division en groupes chronologiques majeurs est préliminaire à ces enquêtes, qui ne peuvent être achevées qu'au fur et à mesure de la définition des types euxmêmes. Pour la plupart, il n'est pas possible d'organiser les types dans un ordre chronologique relatif : au contraire, il faut supposer que plusieurs d'entre eux ont été créés simultanément. Pour cette raison, nous avons décidé d'abandonner les systèmes traditionnels de dénomination qui se servent de lettres et/ou de chiffres. Au lieu de ces solutions, les types coroplastiques de Medma documentés dans le matériel de Calderazzo reçoivent des noms mythologiques, qui permettent aussi un premier regroupement en "familles». Ainsi, des types stylistiquement apparentés entre eux peuvent devenir les Muses ou les Hespérides, etc. La nomenclature, ainsi que l'identification des types eux-mêmes, est actuellement en phase d'élaboration est ne se conclura qu'après l'accomplissement du travail de documentation.

\section{Base de données et exemple de fiche descriptive}

18 L'unité de documentation correspond à l'objet individuel, documenté par une série de photos (fig. 7-9) et par une fiche descriptive bilingue (fig. 10, italien et hongrois). 
Fig. 7 - Statuette de terre cuite de Medma, Rosarno, inv. 40407, vue de face.

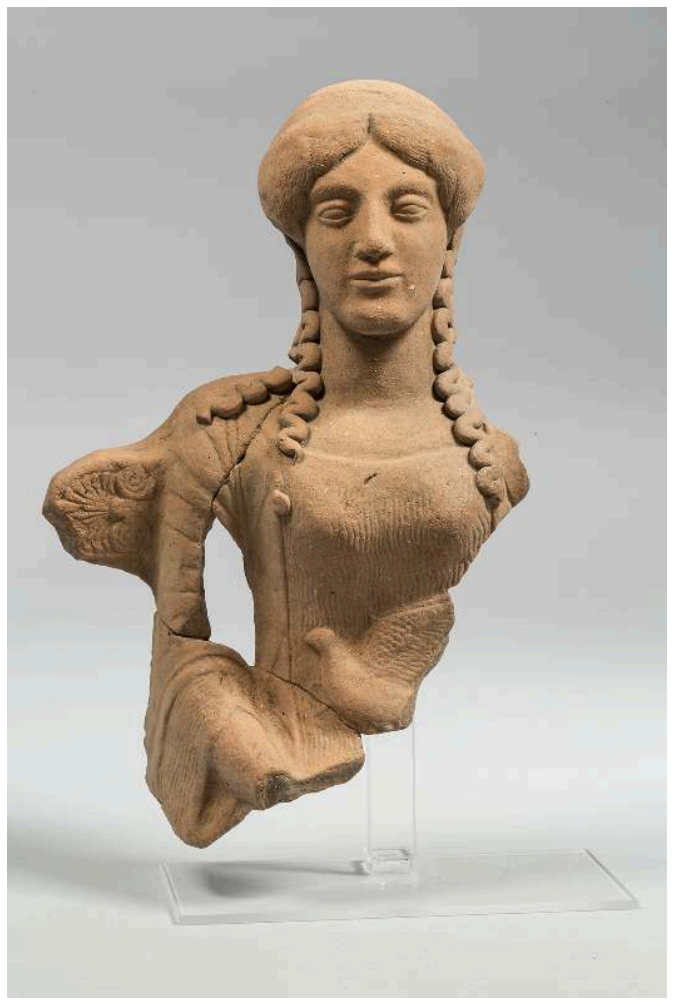

Crédit : L. Mátyus.

Fig. 8 - Statuette de terre cuite de Medma, Rosarno, inv. 40407, vue de profil.

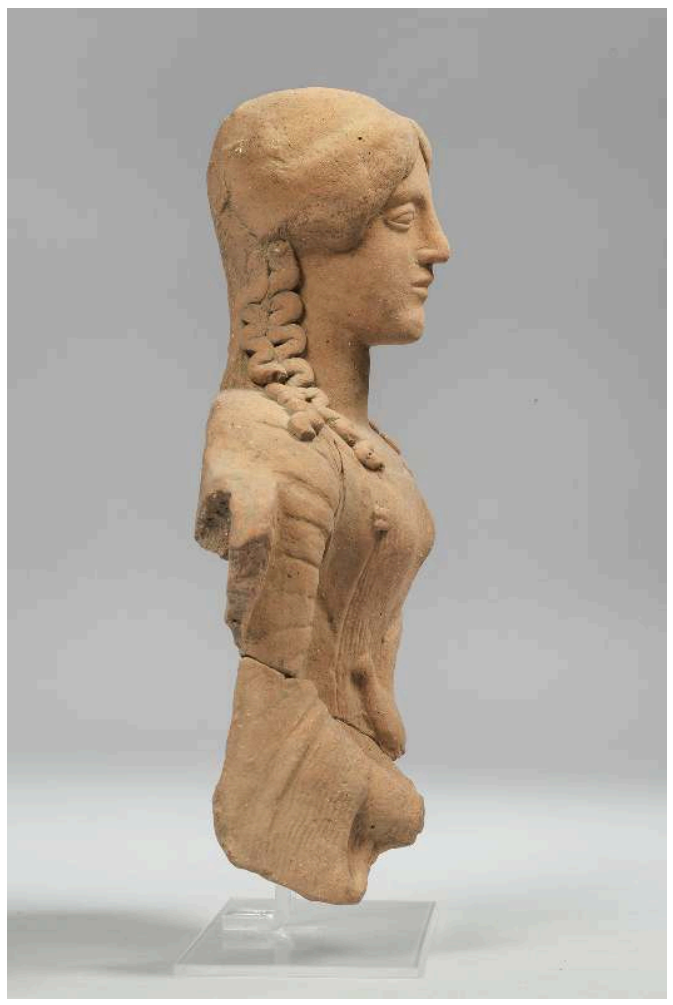

Crédit : L. Mátyus. 
Fig. 9 - Statuette de terre cuite de Medma, Rosarno, inv. 40407, détail.

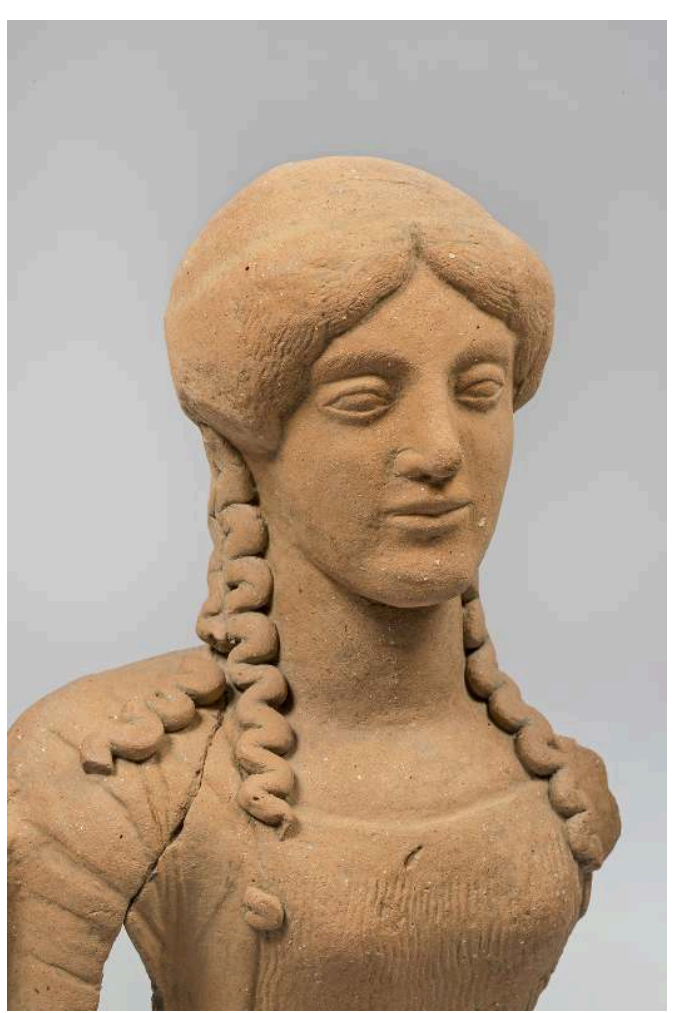

Crédit : L. Mátyus.

Fig. 10 - Exemple de fiche descriptive.

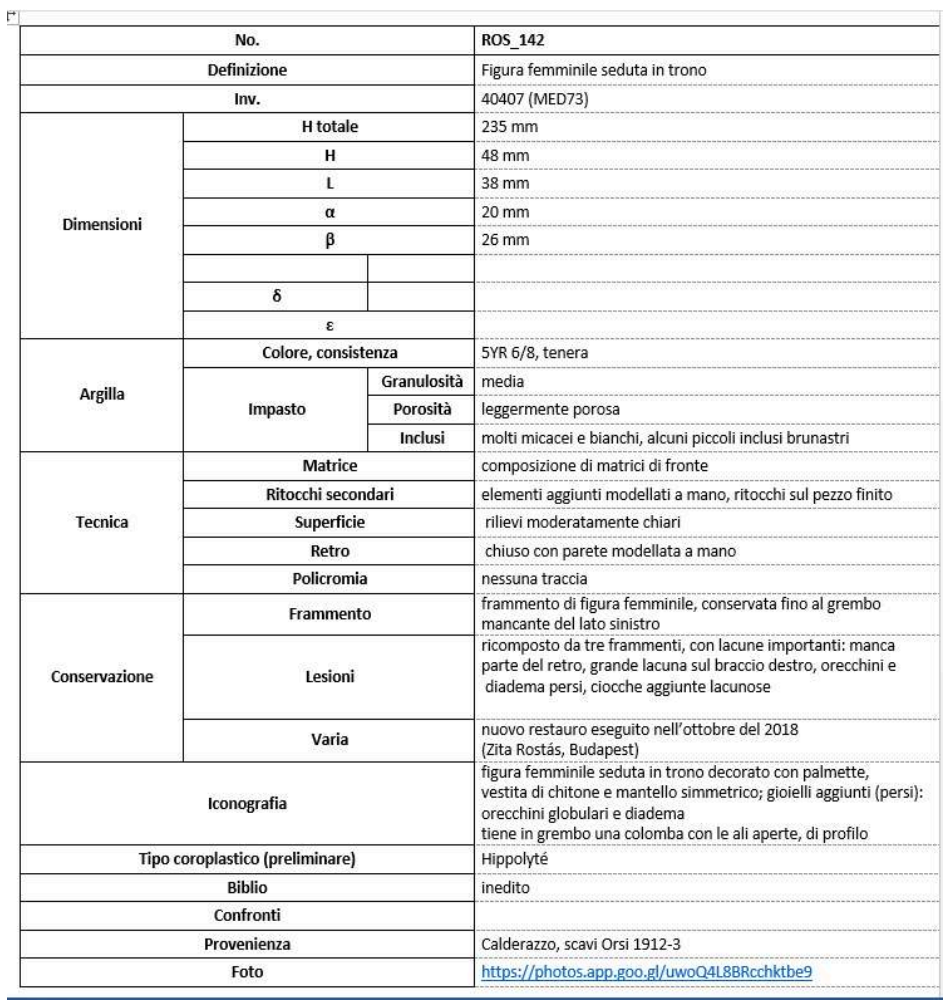

Crédit : Á. Bencze. 
19 tableaux Excel et de fichiers de photos, partiellement en ligne. Le développement d'un support informatique approprié est prévu pour l'an 2020, ce qui permettra le travail contemporain en ligne de plusieurs utilisateurs, ainsi que la publication en réseau ou sur support électronique d'une base de données en format moderne. Au demeurant, notre base de données est déjà apte à servir de point de départ pour différentes orientations de recherches, auxquelles peuvent s'associer encore d'autres, comme les recherches topographiques, la publication systématique des autres classes de documents du sanctuaire de Calderazzo, la documentation de la production coroplastique des autres sites avec la perspective de dresser un cadre plus clair des pratiques cultuelles et des connexions de la cité entre le VI et le IV ${ }^{\mathrm{e}}$ siècle av. J.-C.

20 La documentation photographique revêt une importance particulière, puisque c'est surtout celle-ci qui est à la base des analyses stylistiques, donc du moyen d'interprétation décisif. Pour cette raison, nous tendons à relever et à documenter le plus possible des qualités plastiques de chaque objet. La séquence photographique consacrée à une statuette se compose d'au moins trois, mais possiblement de quatre vues, chacune photographiée avec une série d'éclairages différents (fig. 11).

Fig. 11 - Travail de documentation photographique au Musée National de Reggio Calabria.

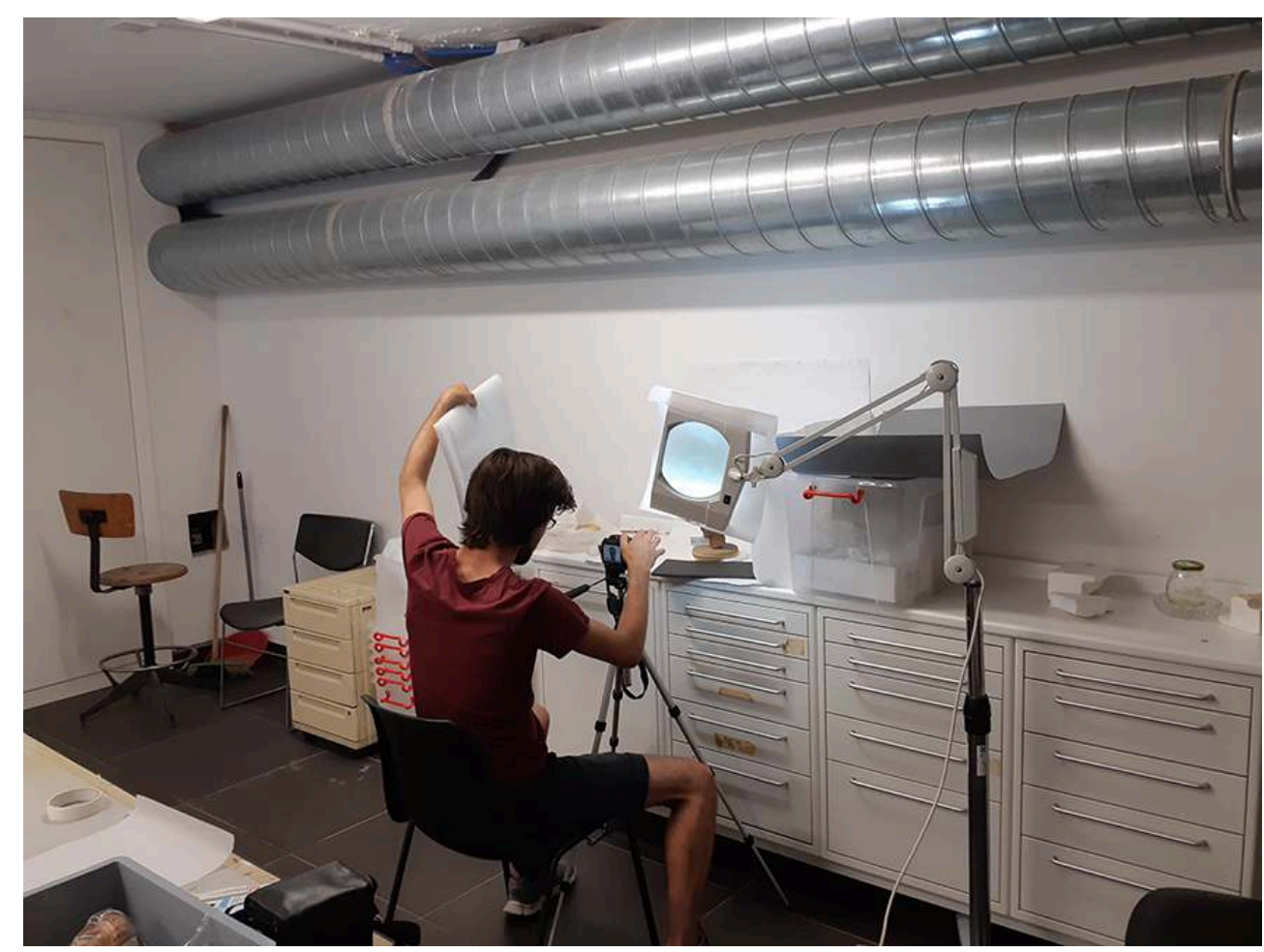

Crédit : Á. Bencze.

21 Afin d'obtenir une documentation visuelle encore plus complète et utile, à partir de la campagne de 2019 nous avons adopté aussi la technique du relevé photogrammétrique, grâce à l'assistance et à la direction professionnelle de Franco Prampolini, professeur d'architecture à l'université de Reggio Calabria. (fig. 12). Dans sa forme définitive en ligne, notre base de données contiendra des modèles numériques en 3D d'une sélection abondante de pièces documentées.

Chronique des activités archéologiques de l'École française de Rome , Italie du Sud 
Fig. 12 - Travail de documentation : relevé photogrammétrique, Rosarno.

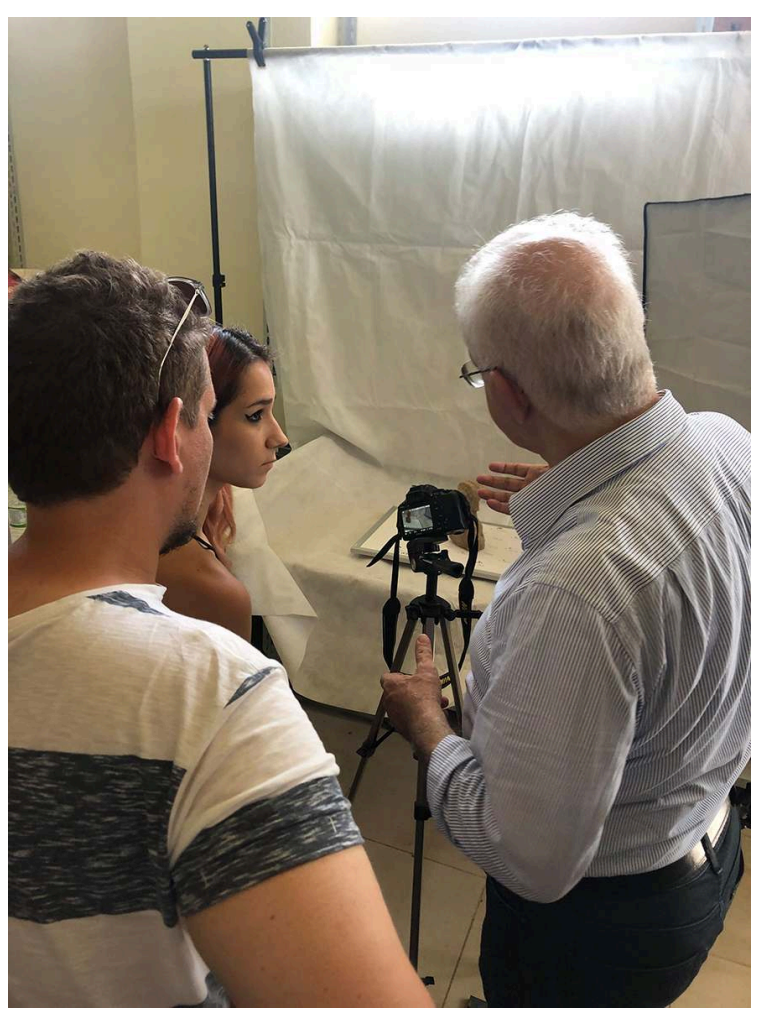

Crédit : Á. Bencze.

\section{Restauration et exposition de 9 terres cuites au Musée des Beaux-Arts de Budapest}

22 Dans le cadre de ce projet, en septembre 2018, un lot de 9 terres cuites votives de Medma est arrivé au Musée des Beaux-Arts de Budapest, prêté par la Surintendance ABAP responsable du Musée de Rosarno. À la suite d'une restauration soigneuse exécutée par les restaurateurs du Musée des Beaux-Arts (fig. 13-17), elles étaient en exposition temporaire, jusqu'au mois d'août 2019 (fig. 18). L'exposition est complétée par une guide en ligne, accessible en hongrois, anglais et italien sur le site du Musée, paru aussi en version imprimée ${ }^{14}$. 
Fig. 13 - Étapes de la restauration, 1. Désassemblage de la vieille recomposition.

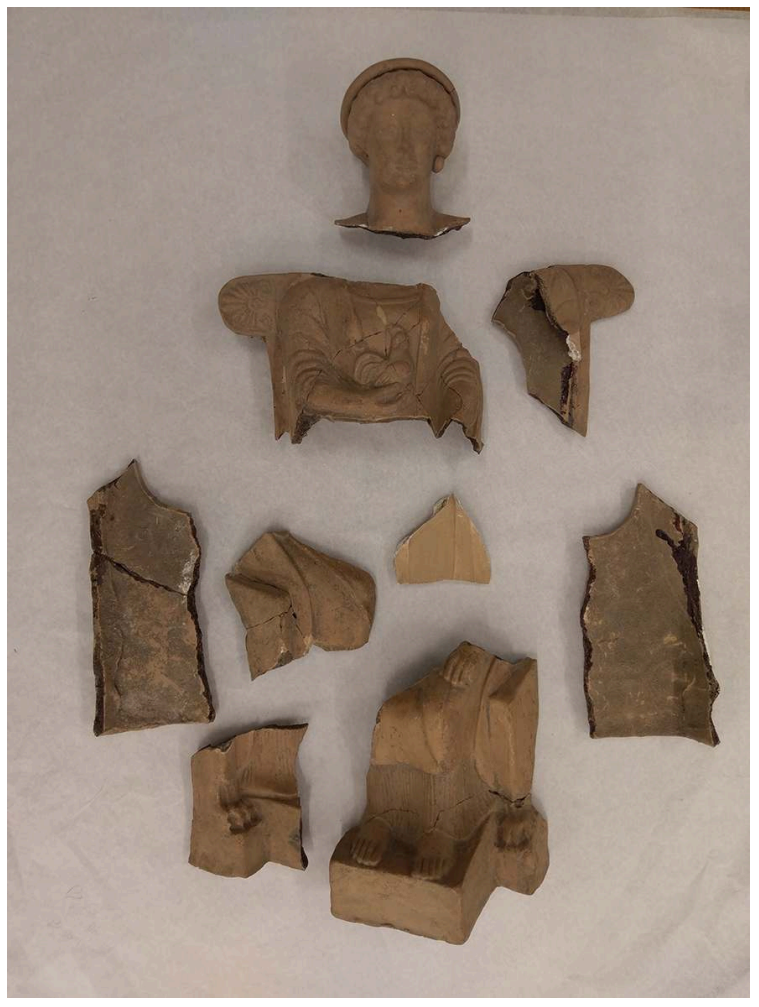

Crédit : Z. Rostás.

Fig. 14 - Étapes de la restauration, 2. Nettoyage des surfaces de terre cuite antique.

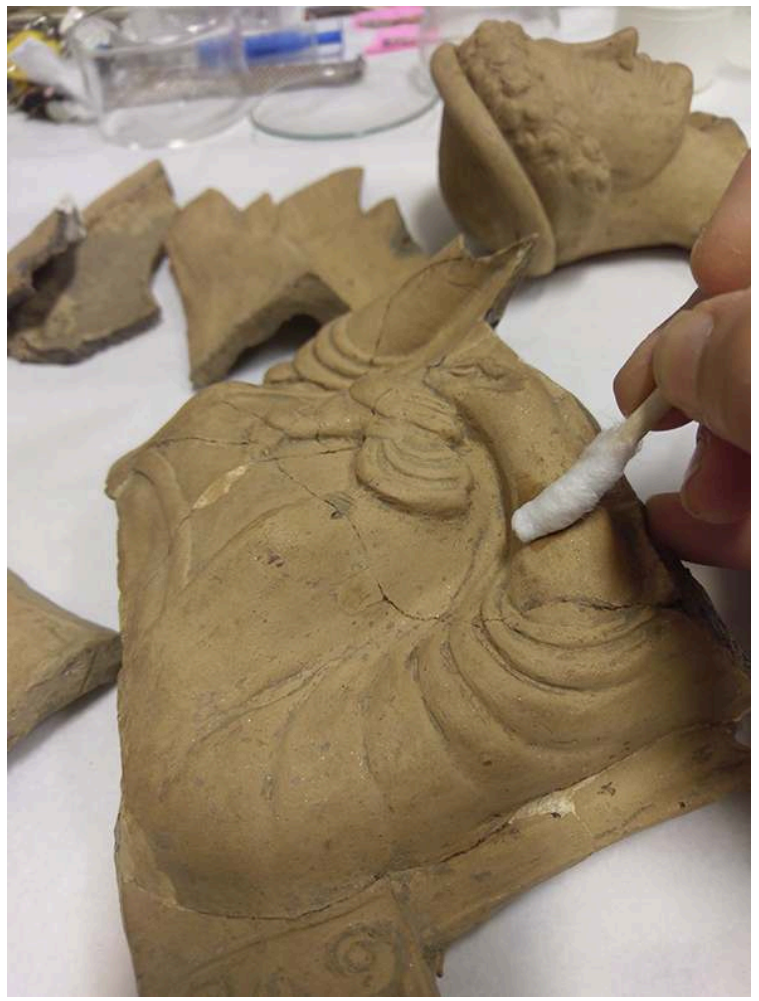

Crédit : Z. Rostás. 
Fig. 15 - Étapes de la restauration, 3. Nettoyage de la surface de l'ancien ajout en plâtre.

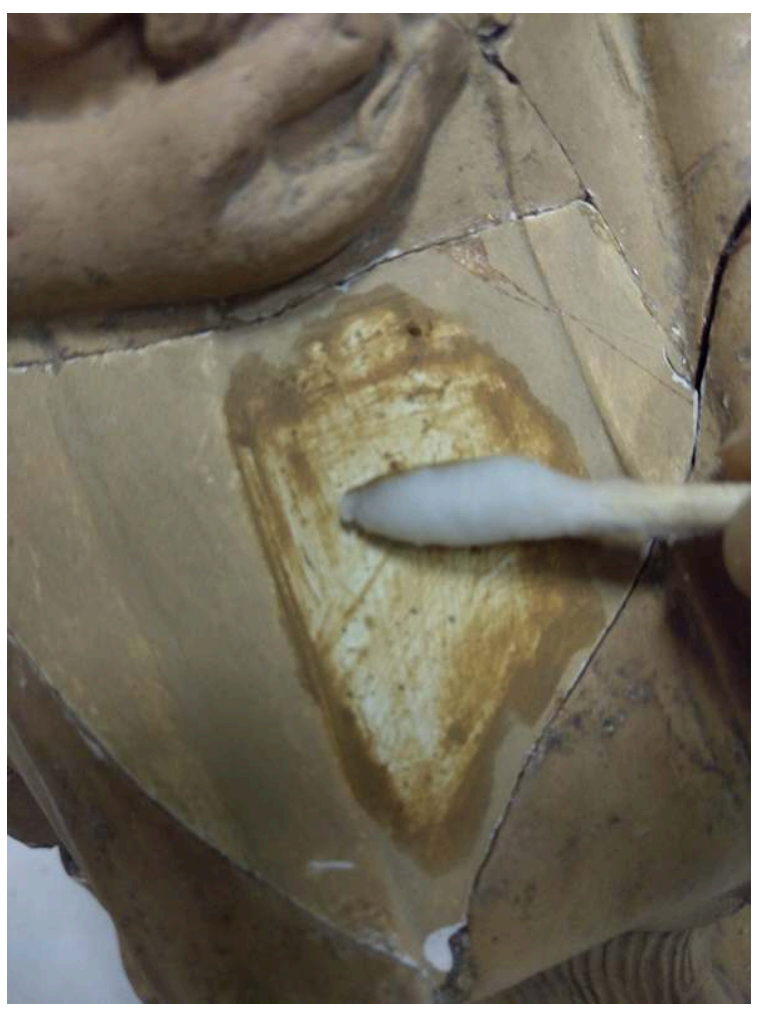

Crédit : Z. Rostás.

Fig. 16 - Étapes de la restauration, 4. Réassemblage.

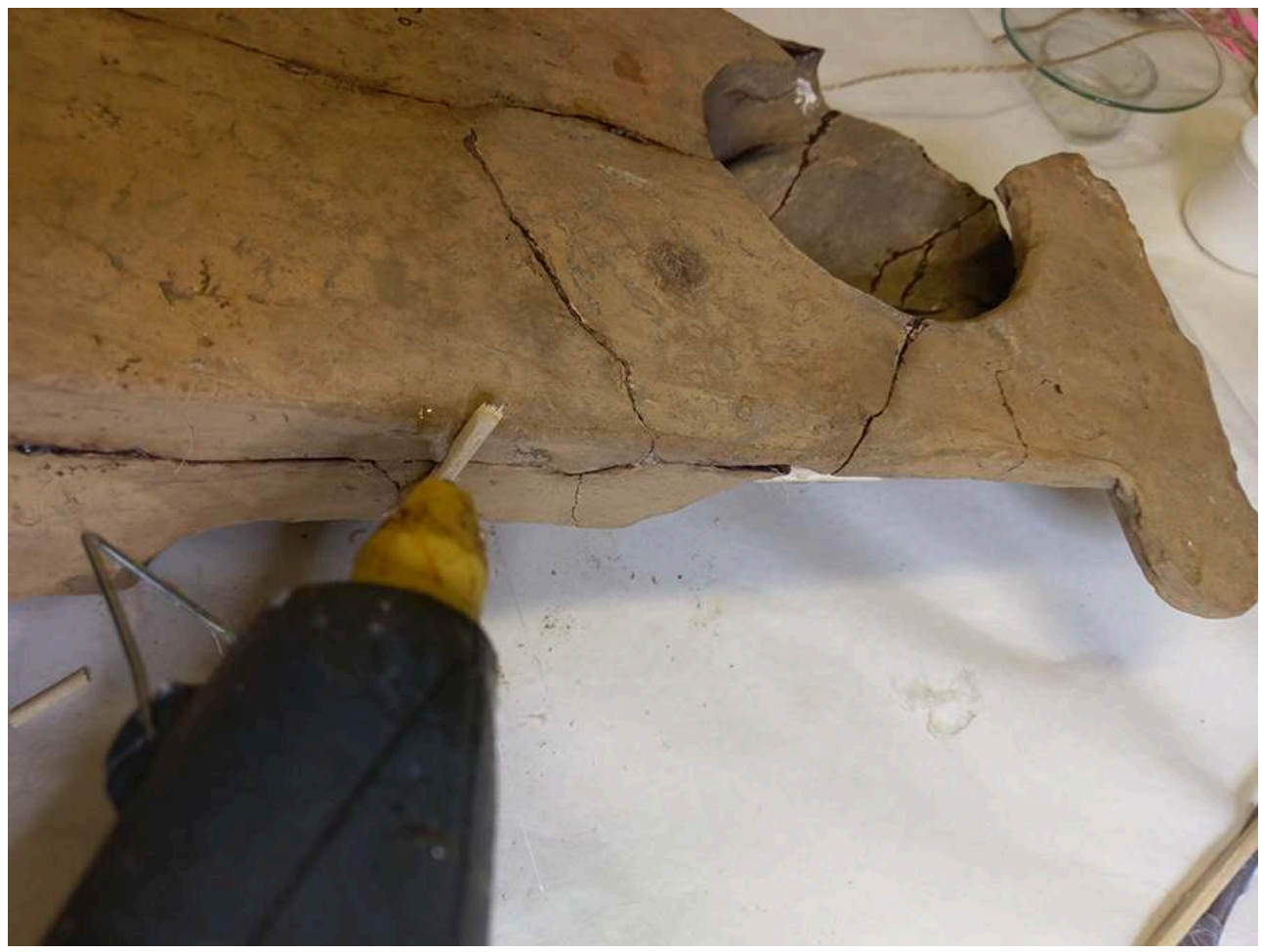

Crédit : Z. Rostás.

Chronique des activités archéologiques de l'École française de Rome , Italie du Sud 
Fig. 17 - Étapes de la restauration, 5. La pièce réassemblée, avant la teinture des ajouts en plâtre.

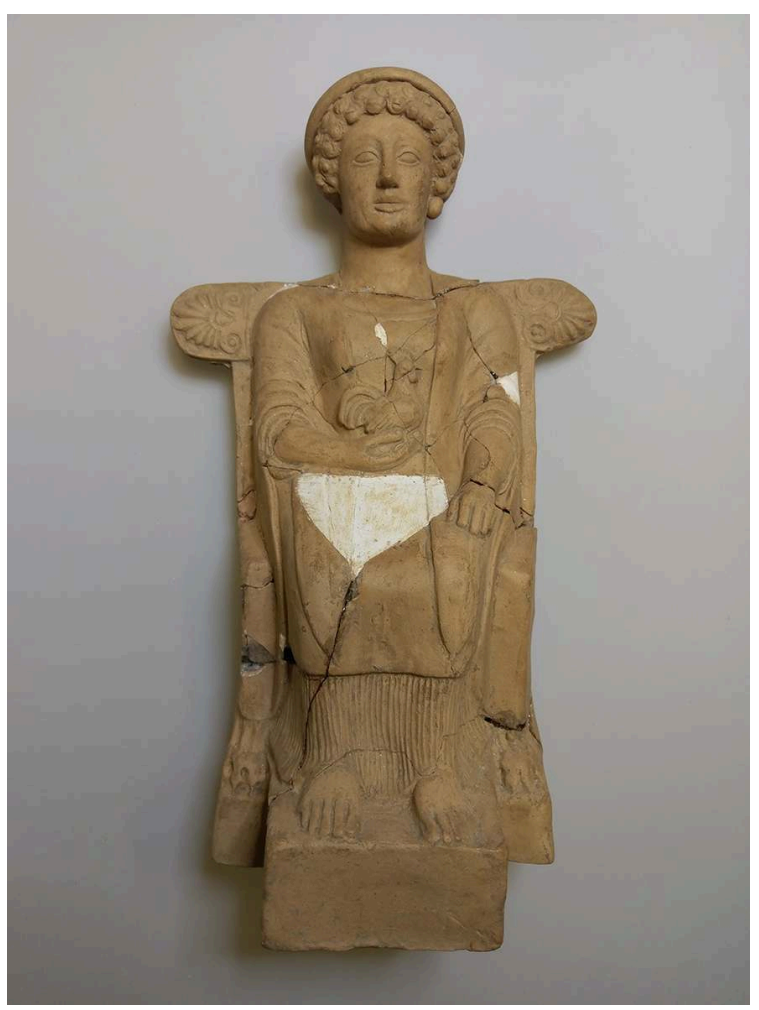

Crédit : Z. Rostás.

Fig. 18 - Statuette de Medma restaurée, en exposition à Budapest.

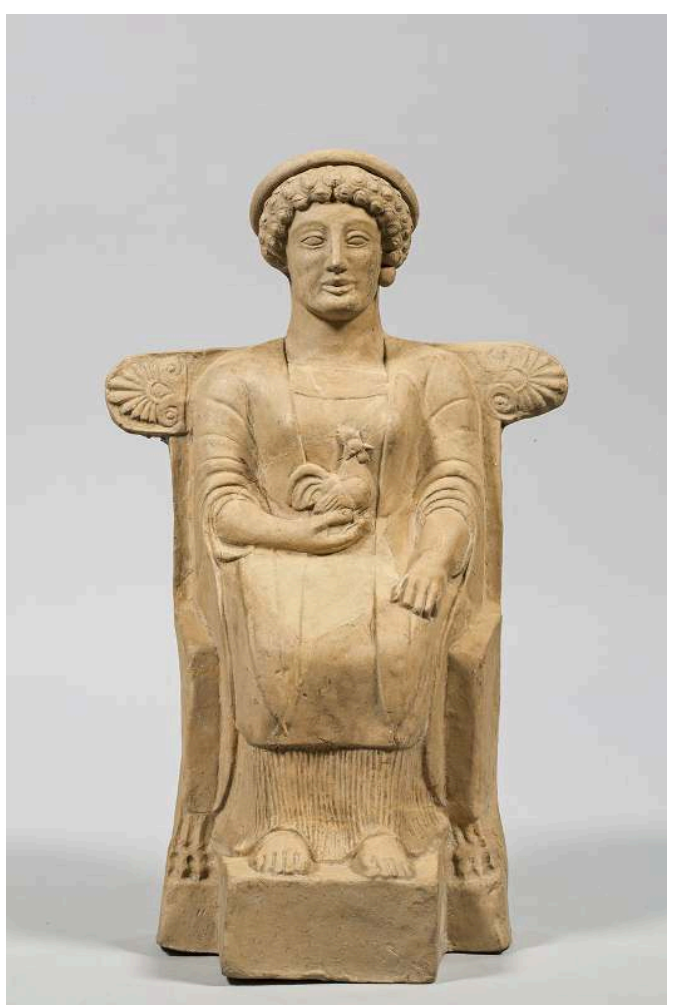

Crédit: L. Mátyus. 


\section{BIBLIOGRAPHIE}

Bencze 2019 = Á. Bencze, Sanctuaries of Medma. Exhibition of the collection of classical antiquities, Fall 2018 - Summer 2019, Budapest, 2019.

Doepner 2007 = D. Doepner, Zur medialen Funktion von Terrakottastatuetten in griechischen Heiligtümern. Ein Befund in Medma (Rosarno), dans C. Frevel, H. von Hesberg (dir.), Kult und Kommunikation. Medien in Heiligtümern der Antike, Wiesbaden, 2007, p. 311-347.

Doepner et al. 2015 = D. Doepner, J. Schulz, A. Skolnik, Terrakottafiguren aus Medma (Rosarno) und ihre digitale Präsentation in Arachne, in Kölner und Bonner Archaeologica 5, 2015, p. 259-268.

Miller 1983 = R. Miller, The terracotta votives from Medma: cult and coroplastic craft in Magna Graecia, Diss. Univ. Michigan, Ann Arbor, 1983.

Miller Ammerman $1985=$ R. Miller Ammerman, Medma and the exchange of votive terracottas, dans Papers in Italian Archaeology, 4, 1985, p. 5-19.

Miller Ammerman $1990=$ R. Miller Ammerman, Terrecotte votive: evidenze di culto e contatto culturale in Magna Grecia, dans Scienze dell'Antichità, 2-4, 1989-1990, p. 353-362.

Muller 1997 = A. Muller, Description et analyse des productions moulées. Proposition de lexique multilingue, suggestions de méthode, dans A. Muller (dir.), Le moulage en terre cuite dans l'Antiquité. Création et production dérivée, fabrication et diffusion, Lille, 1997, p. 437-463.

Orsi 1902 = P. Orsi, Rosarno (Medma?). Scoperte di terrecotte, dans NSc, 1902, p. 47-48.

Orsi 1913a = P. Orsi, Scavi di Calabria nel 1913, dans NSc, 1913, p. 4-54.

Orsi 1913b = P. Orsi, Rosarno (Medma?). Esplorazione di un grande deposito di terrecotte ieratiche, dans NSc, Suppl., 1913, p. 55-144.

Orsi 1917 = P. Orsi, Rosarno, campagna del 1917, dans NSc, 1917, p. 37-67.

Orsi 1928 = P. Orsi, Medma-Nicotera. Ricerche topografiche, dans Campagne della Società Magna Grecia (1926-1927), Rome, 1928, p. 31-61.

Paoletti 1981 = M. Paoletti, Contributo al corpus delle terrecotte medmee e Carta archeologica di Rosarno, dans M. Paoletti, S. Settis (dir.), Medma e il suo territorio. Materiali per una carta archeologica, Bari, 1981, p. 47-92.

Paoletti 1982 = M. Paoletti, Arule di Medma e tragedie attiche, dans A ${ }_{50}$ APXAI. Nuove ricerche e studi sulla Magna Grecia e la Sicilia in onore di P.E. Arias, Pisa, 1982, p. 371-392.

Paoletti 1990 = M. Paoletti, Medma ed Hipponium: gli scavi di Paolo Orsi ai primi del Novecento e le indagini odierne, dans Annali dei Musei Civici di Rovereto, Sezione Archeologia, Storia e Scienze Naturali, 6, 1990, p. 133-162.

Paoletti 2014 = M. Paoletti, Rosarno: l'area sacra di contrada Calderazzo. Scavi Orsi (1912-1913), dans M.T. Iannelli, C. Sabbione (dir.), Le spose e gli eroi. Offerte in bronzo e in ferro dai santuari e dalle necropoli della Calabria Greca, Vibo Valentia, 2014, p. 71-138.

Sabbione 1981 = C. Sabbione, Scavi a Rosarno dal 1977 al 1980: note preliminari, dans M. Paoletti, S. Settis (dir.), Medma e il suo territorio. Materiali per una carta archeologica, Bari, 1981, p. 93-119. Santuari della Magna Grecia in Calabria 1996 = E. Lattanzi et al. (dir.), Santuari della Magna Grecia in Calabria. I Greci in Occidente, Naples, 1996. 
Taccuini Orsi I = P. Orsi, I Taccuini I, édité par G. Lamagna et G. Monterosso, Rome, 2018.

\section{NOTES}

1. Outre la grande série de publications fondamentales de P. Orsi, comme Orsi 1909, Orsi 1913a, Orsi 1913b et Orsi 1928, voir les synthèses de Paoletti 1981, Miller Ammerman 1985, Miller Ammerman 1990, Paoletti dans Santuari della Magna Grecia in Calabria 1996, p. 91-92, 95-97 et 99-111, et Paoletti 2014.

2. Pour une introduction générale à la problématique, voir aussi Bencze 2019.

3. Orsi 1913b. Cf. Orsi 1902, Orsi 1917. Pour les questions concernant la reconstruction topographique des découvertes d'Orsi voir surtout Paoletti 1981, Paoletti 2014 et Iannelli, dans Santuari della Magna Grecia in Calabria 1996, p. 93-94.

4. Cf. Paoletti 1990 et Paoletti dans Santuari della Magna Grecia in Calabria 1996, p. 99.

5. Salvatore Settis en 1964 et 1966, Maurizio Paoletti et Claudio Sabbione dans les années 1970, cf. Sabbione 1981, Paoletti 1981 et Paoletti 2014. Des nouvelles fouilles ont été exécutées par M.T. Iannelli en 2014 et par la Surintendance ABAP, sous la direction de F. Sudano en 2018.

6. Paoletti 2014. Les fouilles de 2014 en ont identifié la continuation, hypothèse confirmée successivement par les fouilles de 2018 qui ont mis en lumière les structures d'un temple.

7. Miller 1983 ; Miller Ammerman 1985 ; Miller Ammerman 1990.

8. Doepner 2007 ; Doepner et al. 2015.

9. Selon le témoignage de Doepner et al. 2015 et la description de projet sur le site de l'Université de Cologne (https://archaeologie.uni-koeln.de/35565.html) les auteurs de cette tentative de documentation n'ont recensé que 600 pièces environ, dont ils ont inséré dans la base de données « Arachne » 163 exemplaires, conservés à Reggio Calabria, presque tous exposés et déjà publiés. Quant au travail de Rebecca Miller, une thèse de doctorat soutenue à l'Université du Michigan en 1983, elle est restée inédite et n'est accessible que sur microfiche. Sa typologie est cependant incomplète et illustrée d'une façon qui ne permet pas d'évaluer la validité des analyses de l'auteur.

10. Les notes manuscrites de Paolo Orsi, les « Taccuini » sont pour partie parus en édition critique (I Taccuini I, 2018), pour partie encore à paraître ou à consulter dans les archives. Les recherches topographiques combinées avec la consultation de ces documents pourraient apporter dans l'avenir des résultats significatifs.

11. Cf. Santuari della Magna Grecia in Calabria 1996, p. 118-126. Sur classe particulière des modèles d'autel décorés de scènes figurées en relief, voir Paoletti 1982.

12. L'importance particulière des analyses interdisciplinaires pour le cas spécifique des terres cuites votives de Medma fut déjà reconnue par R. Miller, sans que sa recherche ait pu profiter d'analyses chimiques systématiques du matériel : cf. Miller Ammerman 1985.

13. Pour la terminologie utilisée, je renvoie à Muller 1996.

14. http://antik.szepmuveszeti.hu/antik_gyujtemeny/blog/?p=2801; Bencze 2019. 


\section{INDEX}

Index géographique : Medma

Mots-clés : Terres cuites votives, Grande Grèce, Medma, Locres, pratiques rituelles, typologie, analyse stylistique

institutions Université Catholique Péter Pázmány, Budapest ; École française de Rome ; Museo Archeologico Nazionale di Reggio Calabria (MArRC) ; Soprintendenza Archeologia, Belle Arti e Paesaggio per la città metropolitana di Reggio Calabria e la provincia di Vibo Valentia (ABAP RC-VV) ; Szépművészeti Múzeum, Budapest

\section{AUTEUR}

\section{ÁGNES BENCZE}

Université Catholique Péter Pázmány, Budapest 